\title{
INTELLIGENT MANUFACTURING SYSTEMS IN CONCEPT OF DIGITAL FACTORY
}

This paper deals with selected areas of concept of Zilina Intelligent Manufacturing System (ZIMS), which is developed by the Faculty of Mechanical Engineering in collaboration with CEIT and other faculties of the University of Zilina. Main research areas realized in the framework of ZIMS cover the basic processes in the entire product lifecycle from beginning to end (PLM - Product Lifecycle Management). In this paper two of these areas will be described - manufacturing system design using progressive digital technologies and utilization of automation and robotics in intelligent manufacturing systems.

Keywords: Intelligent Manufacturing Systems, Zilina Intelligent Manufacturing System, robotics, simulation, virtual 3D models, optimization of production systems.

\section{Introduction}

Nowadays companies must be sufficiently flexible with respect to increasing diversity and changes in customer requirements and needs, so that they can stay competitive in the current dynamic and turbulent environment. The time becomes a key factor, what is reflected in the need for constant shortening of product innovation cycles and consequently in the need for shortening of processes and systems innovation cycles [1]. Intelligent Manufacturing Systems represent the actual answer to the solution of the above mentioned problems.

Intelligent Manufacturing Systems are socio-economic systems with the ability to autonomously identify system changes and impulses from the environment, their causes and to use the obtained knowledge for self-learning, adapting and responding to all changes of the surrounding environment in a way similar to human response.

The implementation and operation of intelligent manufacturing systems requires highly qualified specialists able to design and to apply new methods and technologies based on artificial intelligence and utilization of progressive digital technologies, able to increase the quality, flexibility and efficiency of designed manufacturing systems [2].

The concept of Zilina Intelligent Manufacturing System (ZIMS) developed by the University of Zilina in collaboration with CEIT solves several research areas:

- Intelligent Manufacturing Systems

- Digital Factory Technologies

- Design and simulation of manufacturing processes and systems
- Virtual and augmented reality technology

- Virtual design and testing of products

- Bionics and bionics principles exploitation

- Reverse engineering and Rapid Prototyping

- Holonic production control

- Intelligent transportation and handling systems

- Artificial Intelligence

- Automation and Robotics

\section{Methodology of interactive manufacturing systems design}

At the University of Zilina a new approach to manufacturing systems' design was developed within the framework of the ZIMS and the concept of digital factory. This new approach is based on using of progressive technologies like reverse engineering, 3D modeling, interactive design and augmented reality.

The manufacturing system design process is carried out in the following phases (Fig. 1):

1. Preparation and analysis of input data:

The main source of information for manufacturing system design is the database of data from a design and technological preparation of production. The data for a design of production layout shall include the information on:

- products which are going to be produced in the production system (types of products, bills of material, part design parameters, production volumes planned, etc.)

- their production processes (manufacturing and assembly processes, technologies, time standards, etc.)

\footnotetext{
* Martin Krajcovic ${ }^{1}$, Vladimir Bulej ${ }^{2}$, Alzbeta Sapietova ${ }^{3}$, Ivan Kuric ${ }^{2}$

${ }^{1}$ Department of Industrial Engineering,

${ }^{2}$ Department of Automation and Production Systems,

${ }^{3}$ Department of Applied Mechanics, Faculty of Mechanical Engineering, University of Zilina, Slovakia, E-mail: kpi@fstroj.uniza.sk
} 
- resources to be used for their production (machinery, equipment, tools, personnel, transportation and handling equipment etc.).

Key outputs from the input data analysis have to provide an overview of the planned material flow in the production system (material flow analysis) and total need of resources required for implementation of the planned production (production system capacity dimensioning). These data sets form the basis for a proper layout design (optimal layout of machines with respect to material flow) and time plan (balancing the production performance of individual workstations) of the future production system.

\section{3D objects preparation:}

Design and visualization of the production system in a digital environment requires the preparation of 3D models for all components which the production system consists of (production machines and equipment, transport and handling equipment, handling units, storage facilities, auxiliary equipment, etc.). The methodology uses three basic approaches to obtain 3D objects:

- Using the existing model libraries of available software solutions

- Acquisition of new 3D models by using reverse engineering methods - 3D laser scanning (see chapter 3)

- Creation of new models using CAD software (AutoCAD, Microstation, CATIA, etc.)

\section{Manufacturing system modeling:}

The phase of modeling production system (see Chapter 4), includes the design of initial layout which represents the ideal layout of workstations in the production system with regard to the transport relations between workstations and design of a real layout that takes into account all the real constraints in the production system, resulting from respecting the building construction solution, existing engineering networks, links to the logistic chain of the company and service processes.

4. Manufacturing system optimization and visualization:

The interactive design approach is used to optimize a layout configuration (see Chapter 5) which allows monitoring and evaluating of the implemented changes in the production layout immediately after their realization. For this serves a set of analytical tools to help the projection team in determining and evaluating changes. For the interactive visualization and designing of the production system its own solution represented by a planning projection table

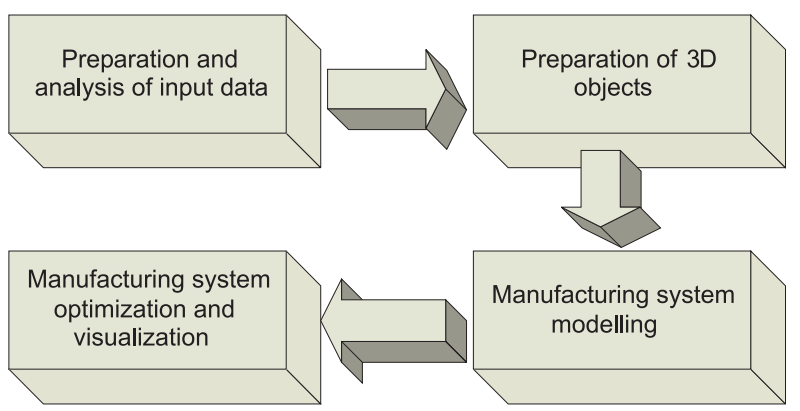

Fig. 1 Methodology of interactive manufacturing system design is used. This table is complemented by a $3 \mathrm{D}$ visualization using augmented reality technologies.

\section{Virtual 3D models preparation by using reverse engineering methods}

In the process of reverse engineering a real object is transformed by the digitization into the computer model. In the case of a manufacturing systems design, 3D digitizing is used for creating models of the production system components (DMU - Digital Mock Up), as well as for digitization of an entire production area (FMU Mock Up Factory). Digitization technology is based on the use of 3D scanners [3]. Obtained data from the scanner are further adjusted by software and the result of these changes is a computer model of the object.

The methodology for creating 3D models of machines and equipment by $3 \mathrm{D}$ scanning is divided into four successive phases: 1. Preparatory phase of 3D laser scanning process consists of:

- selection of real object needed for solution of the problem

- collection of data about a scanned object (object photo-documentation, drawings documentation of the object, technical documentation)

- Scanning plan preparation (preliminary positions of the scanner at the scanning process and positioning of reference points needed for the scanning process)

2. 3D laser scanning phase consists of:

- proper deployment of reference points and suitable location for the scanner

- connection of the scanner with the software equipment and setup of parameters

- launch of 3D laser scanning

3. Phase of editing scanned data:

- import of 2D panoramic photos

- marking references in 2D panoramic photos

- creation of 3D point cloud model

- modification of the model and filtering

4. Phase of editing and modeling scans in CAD system:

- import data into the CAD program

- creation and editing of polygonal network model

- redrawing of polygonal network into the $3 \mathrm{D}$ model

\section{Production system modeling}

In the first phase of modeling the production system an ideal layout of workplaces is designed. In the ideal layout the real space requirements of workplaces, input - output points of the production system or other constraints (e.g. space restrictions) are not taken into account. To create the ideal layout a heuristic algorithm is used. During the optimization process this algorithm minimizes the total transport capacity calculated as the sum of the Euclidean distance and intensity of transport between all workplaces. 


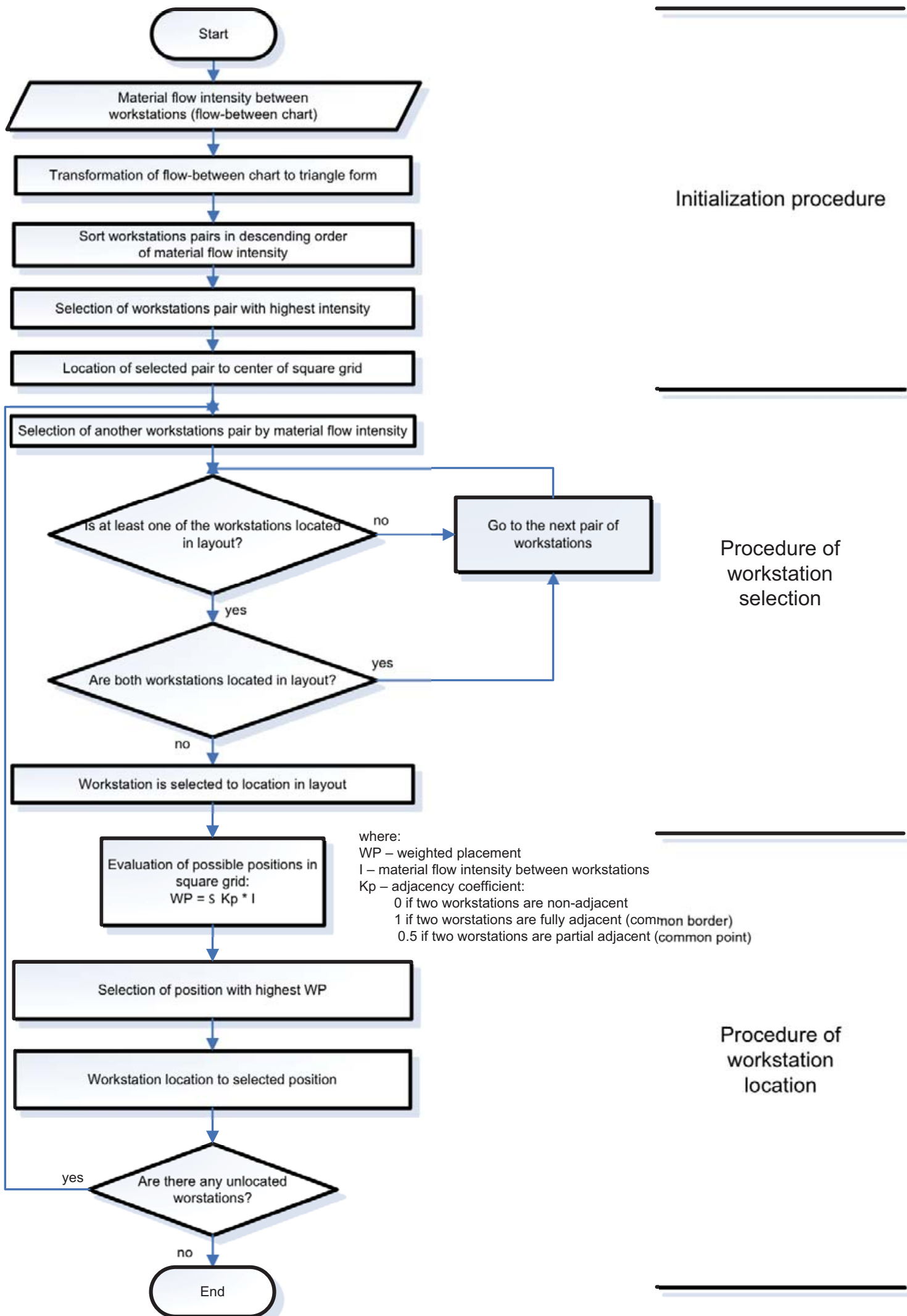

Fig. 2 The algorithm of the ideal layout design 
The algorithm is divided into 3 phases (Fig. 2):

1. Process initialization: Includes processing the input data in the form of a checkerboard table of transport relations, sorting of workplaces pairs (sort from the highest to the lowest transport intensity) and the selection and placement of pairs with the highest intensity into the layout.

2. Workplace selection procedure: Serves for selection of a workplace that will be in the next step assigned to the workplace already placed into the layout.

3. Workstation placement procedure: It serves for a suitable selection of a position of a chosen workplace in the layout considering earlier placed workplaces and relevant transport relations.

In the second phase of modeling the ideal layout is transformed into real (Figs. 3a and 3b), and respects:

- real shape and dimensions of the machine / workplace and workplaces operating areas (represented by 2D / 3D model),

- basic material flow in the production hall and its connection to the material flow between different halls (input, output, stores location),

- transport and material handling needs (handling units in production, transport network and streets dimensioning, cranes paths, etc.),

- placement of building elements (columns, walls) and permanent installations (electricity, industrial gas stations, etc.).

\section{Optimization and visualization of production system}

For the purpose of the layout optimization the approach of interactive team layout design is used which uses as a technical resource its own concept of the projection planning table [4] which has been designed in two technical variants:

1. Projection planning table based on the principle of bottom projection of the image on the semi-transparent surface and interactive control of the objects on the screen using the eBeam system (Fig. 4a).

2. Projection planning table using touch screen (Fig. 4b).
Decisions about changes and adjustment of the layout are realized by a projection team using basic analytical tools which enable continuous evaluation of the consequences of the decisions made by the team on parameters of the production system:

- Sankey diagram

- I - D diagram (Intensity - Distance diagram)

- calculation of the overall transport performance

- triangular method

- analysis of safety equipment service areas

- analysis of safety of operating areas and equipment

Visualization of the projected production system in the 2D view on the area of the projection planning table is accompanied by a $3 \mathrm{D}$ visualization using augmented reality technologies $[5,6]$ which add selected virtual elements / objects into the real environment. Technology of the projection planning table is supplemented by AR virtual 3D models of various elements of the production system and the models of workplaces in the 2D layout serve as markers (signs which identify the correct position and orientation of each 3D object) (Fig. 5a). In the final phase AR technology is also used for visualization of the production system in the real environment of the production hall (Fig. 5b).

\section{Application of industrial robots within the factories}

Industrial robots have a distinct set of capabilities that allow them to perform in industrial environments while also distinguishing them from other specialized robots. A network of complex mechanical gestures, triggered by sensors and computer software enables industrial robots to perform a wide range of tasks: spot and arc welding, picking up and clamping activities, clamping for machining, and the transfer and manipulation of parts are all common applications for industrial robots. As European economies start to recover from the recession, manufacturing companies are seeking ways to ramp up production in a flexible way. One option is to use industrial robots which are now more cost-effective both

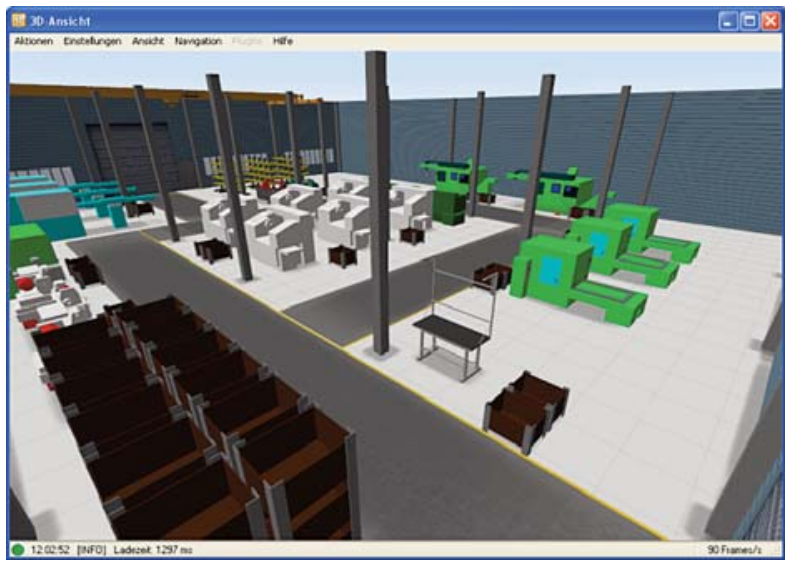

b) $3 D$ layout

a) 2D layout

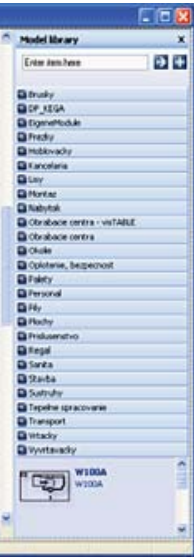

Fig. 3 Real layout of manufacturing system 


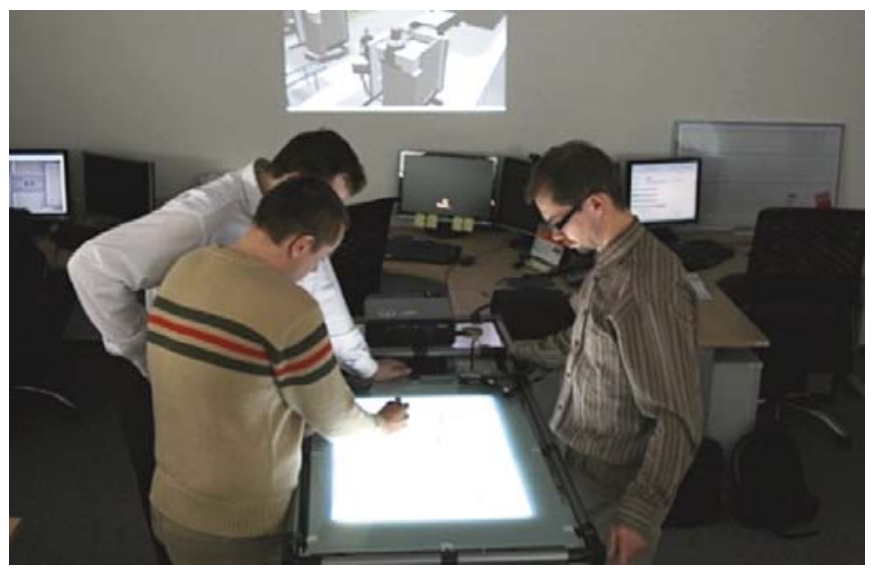

a) Planning table with down projection

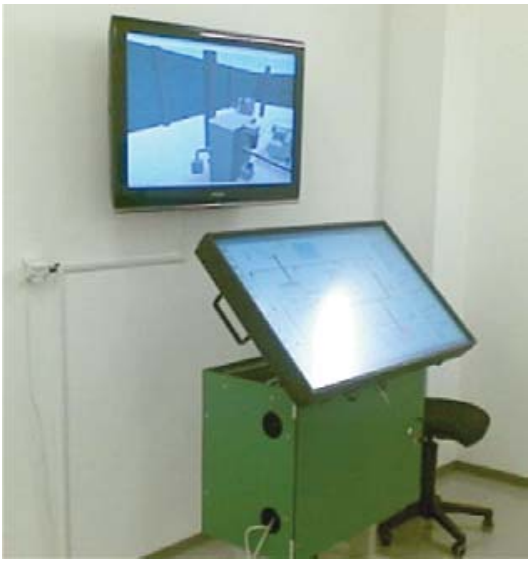

b) Planning table with multitouch display

Fig. 4 Variants of the projection planning table

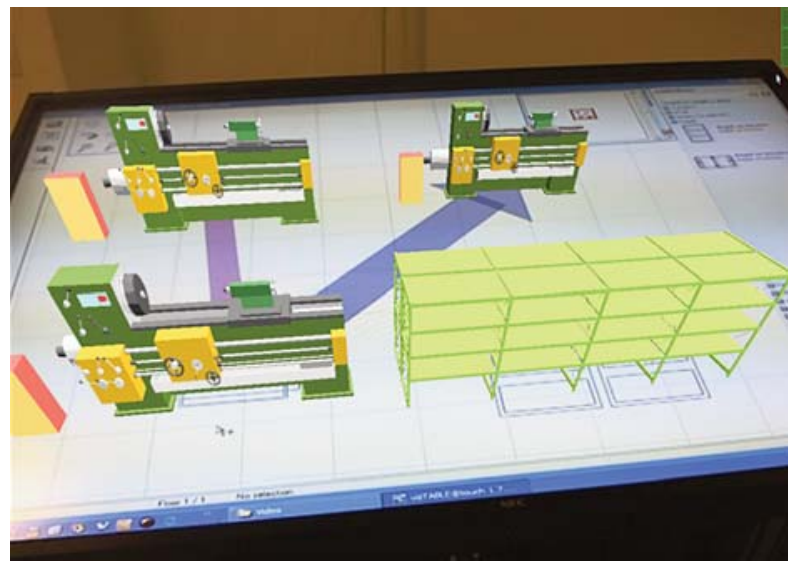

a) Planning table

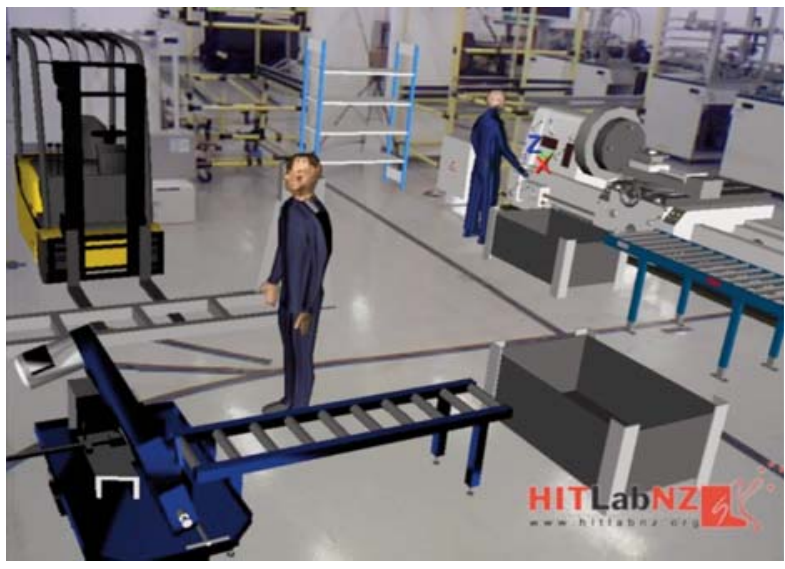

b) Real production hall

Fig. 5 Layout visualization using augmented reality

to purchase and to implement, thanks largely to programming software that is more engineer-friendly.

In field of industrial robots several trends are visible as, for example:

- application of force/torque sensors,

- modular and reconfigurable structure of robots for specific applications,

- increasing of ratio between robot's weight and payload,

- robots are able to carry heavy objects (car chassis),

- special robots for service operations,

- automated assembly and control operations (KUKA Sensibot),

- specialization and differentiation of robots,

- higher resistance within environment with specific conditions (water, dust, etc.),

- grasping of randomly placed objects by machine vision systems,

- cooperation of multi-arm systems,

- system integration (robot with camera system, robot mounted on mobile platform),
- application of robots for technological operations as machining, etc.

For industrial automation the four main types of robot are Cartesian (gantry) robots, SCARA types, anthropomorphic robots that typically have five or six axes, and Delta (parallel kinematic) types. SCARA still leads in the electronics sector, but the speed of the standard arm has improved to rival that of SCARA now. A Cartesian robot more closely matches the motion of a human arm where manual tasks need to be automated. The capabilities of industrial robots can be increased by peripheral devices as feeders, belt conveyors, camera systems, systems for automatic tool changing, index tables etc. [7]. Nowadays the robots have improved properties as higher speed, precision, rigidity, payload and efficiency.

With robots now being simpler to integrate, there is increasing competition among manufacturers to add functionality so they can offer increasingly sophisticated systems. An example is the integration of a vision system into the control system of each 


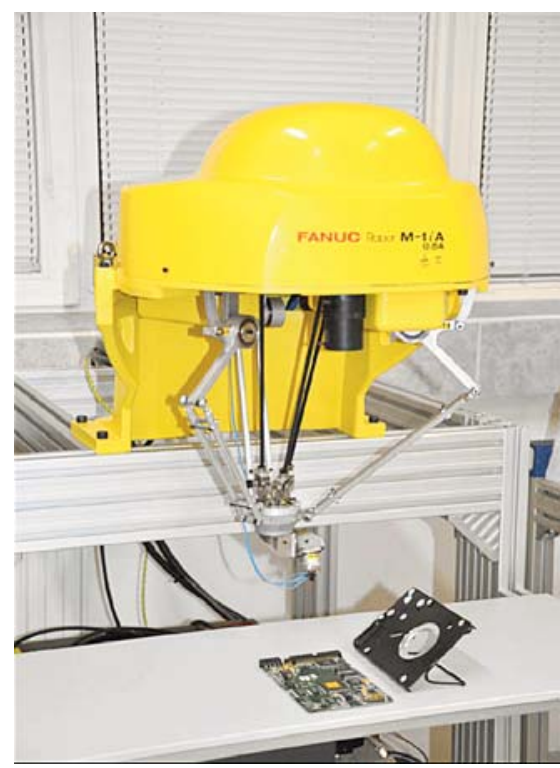

a) Application of delta robot for assembling of electronic device

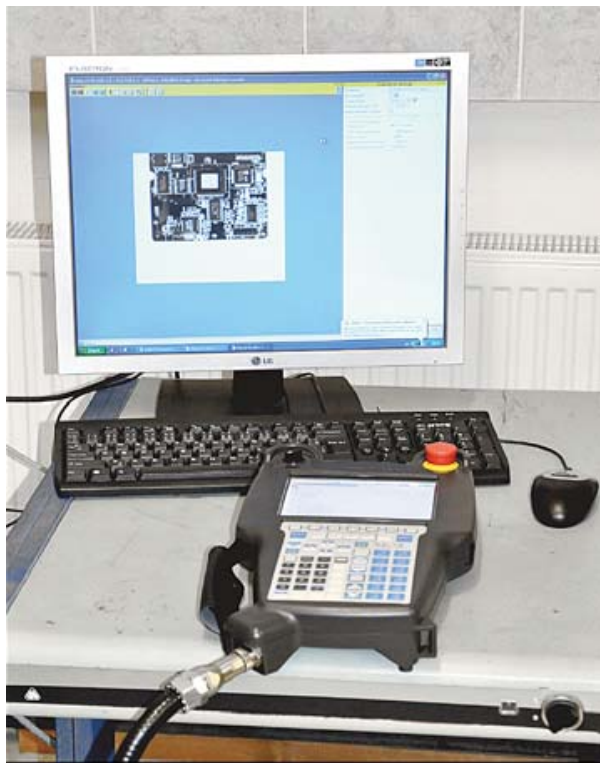

b) Control system include the Vision Tool and so-called teach-pendant

Fig. 6 Application of delta robot FANUC M1-iA with integrated vision system

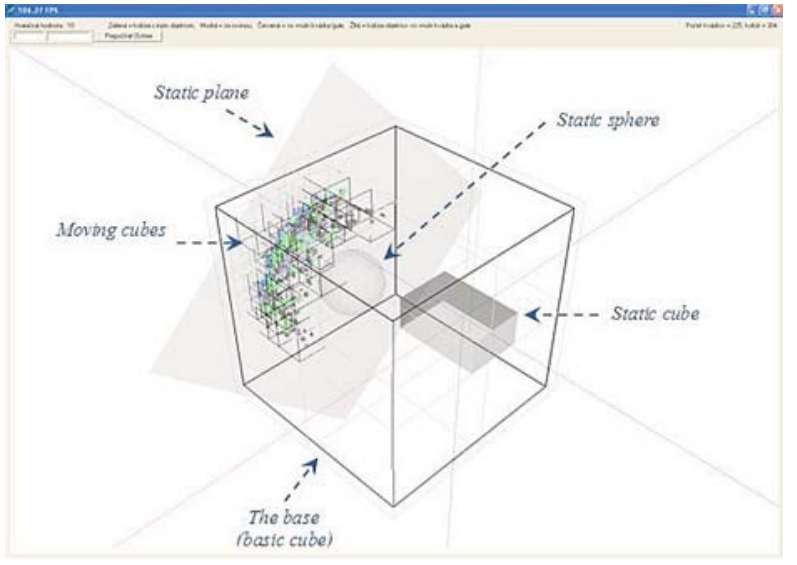

a) Principle of anti-collision system (Department of Automation and Production Systems) [9]

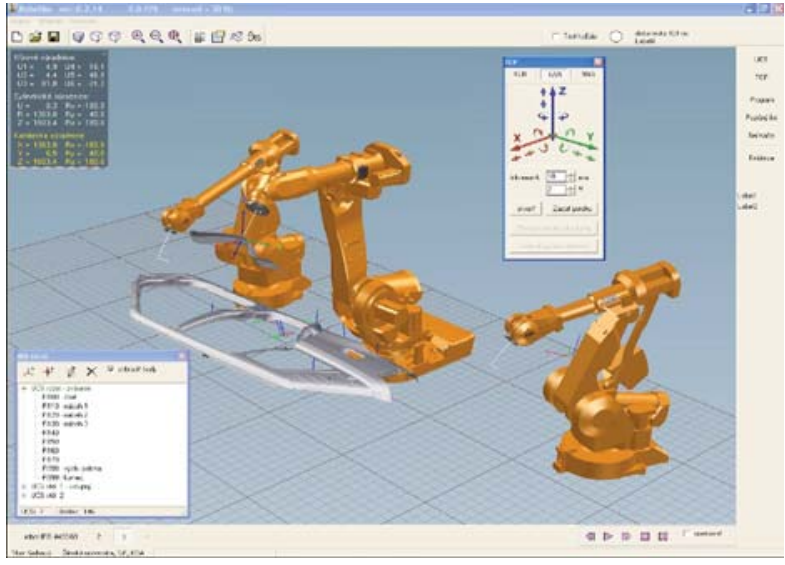

b) Simulation software for collision detection of robotized workplaces within $3 D$ space

Fig. 7 Designed simulation software for collision detection within 3D space

FANUC robots. The designers of robotic cells are pushed to integrate more processes and solve them by only one robot. It means that the robot should have more end-effectors. The best solution is the system of automatic tool changing controlled by pneumatic or electric fixation units (for example made by co. SCHUNK, ATI Automation, etc.).

The Delta robot FANUC M-1iA (Fig. 6a) is a novel lightweight robot for electronics, measuring device and other precision industries [8]. This robot provides a higher productivity for assembly and picking applications, a lightweight mechanism with better cycle times and a unique 6-axes parallel link arm mechanism that allows automate difficult tasks such as complex insertion, gluing and more. The compact and intelligent controller integrated iRVision (Fig. 6b) which can locate and check work pieces for flexible parts feeding applications and other intelligent tasks. This vision system allows four possible processes: normal 2D Vision, depalletizing 2.5 D Vision (includes a calculation of Z-height and some special depalletizing functions), 2D Multiview Vision and Visual Line Tracking 2D Vision. The task of keyboard assembling the numbers of a sloping keyboard is an example of real application. 


\subsection{Collision detection, prediction and avoiding}

Because of still quite a high price of robotic devices, the recent industrial robots are able to modify the realized trajectory and this way to avoid collision with any object within the workspace (Fig. 7a). This avoiding process is done during its movement thanks to application of appropriate sensors and control system. Their deployment is particularly suitable for the tasks which require immediate correction of the track in response to a sudden, not expected changing of the real working environment.

Under the term "anti-collision control system" we understand any software, hardware, seeking to avoid collisions on real machines. In general these systems can operate in two modes [9]:

Off-line mode - in this case we apply the specialized simulation software (for example: simulation software shown in Fig. 7b). There is created or loaded a 3D virtual model of handling device, endeffector, manipulated objects, peripheral devices and any other components placed in the real robot workspace. The control system must continuously detect the collisions during the run of simulation. If there arise any collisions the simulation software shows them. Then the operator can modify the original program and repeat the loop until a collision-free trajectory is obtained.

On-line mode - is an active approach. Directly during the robot movement the control system tries to avoid a collision with any objects in real time. In this case it is necessary to have enough numbers and appropriate types of sensors. The best way is to combine several different types of sensors as, for example, tactile sensors, ultrasound or infrared sensors and cameras. On-line systems don't need the model of environment and they can work with or without the model.

\section{Mobile robotics and its integration within the factories}

A separate area in robotics called mobile robotics was established during the last period. This area is focused on the development of suitable mobile robotic devices, methods, special algorithms and finding their appropriate application in real world. Generally, the mobile robot (MR) is a complicated mechatronic cognitive system. Selection of appropriate navigation method in the workspace is one of the fundamental problems which is solved by the design process of all mobile robots [10].

The reasons for mobile robots development and their application are, for example, safety level of processes, exploration of unknown terrain, inaccessibility and unavailability, reliability. Increasing the safety level of processes, reliability and continuous growing of production efficiency are also the very important reasons for their application in industry. They can be applied as a main transport system within the factory, they can co-operate with standard industrial robots in robotic cells and they can be used also as cleaning or other kind of service devices. According to their application they have different level of autonomous behavior. In the highest level we can speak about autonomous mobile robots
(AMRs). Each AMR is composed of a mechanical subsystem (undercarriage), a sensoric subsystem (internal and external sensors), a control subsystem (control of all subsystems of AMR), a communication subsystem (data transfer and communication with operator). With respect to the possibility to execute and record the realized trajectory, it is necessary to create a suitable mathematical description of the mobile robot, movement method and model of environment.

Examples of mobile robots applied in the industrial environment are represented by a mobile robot OmniMove with a mounded robotic arm made by co. KUKA, robotic system AutoStore (made by co. Swisslog) for manipulation within the warehouse, mobile robot equipped with omnidirectional wheels Vetex, etc.

\subsection{Control system suitable for mobile robots in industry}

The control system together with control algorithms are the basic parts of all mobile robots. The hardware of control system must be able to process any input information from the sensoric, communication and navigation subsystem in qualitative and quantitative meaning too. The main control program or some subroutines must handle with raw data - their preprocessing, analyzing and finding of adequate reaction of actuators (actuated wheels and actuated technological tools). The mobile robot autonomy significantly affects the final configuration of the designed control system. Roughly speaking, the mobile robot control system can be based on: - personal computer,

- industrial computer (IPC - Industrial PC) or

- microcontroller.

The control system based on a standard personal computer is not very suitable for mobile robots applied in a factory [10]. This kind of applications requires the control system with some special characteristics (resistance to different temperature, humidity, dust and vibrations, electric noise, etc.). All these requirements and some others can be met using the control system based on IPC or based on the microcontroller (for example Atmel AT MEGA). For exploring the surrounding environment the system of proximity sensors is the most common. Tactile sensors are used only as a backup safety element to activate the emergency stop. Only the combination of different processing methods and the application of various types of sensors can increase the quality of output information.

\subsection{Navigation of mobile robots}

By robot navigation within a space with obstacles, the goal is to find a collision-free path of a robot from the starting to the target (goal) position. Navigation strategies can be classified into several groups from the viewpoint of a method of sensors' data processing, representation and type of environment and level of path planning. At the bottom is the pure reactive control oriented only on obstacles avoidance when the nearest space surrounding 
the robot is scanned. Next level is local navigation which solves also localization. When the robot knows the environment and has its own map, we can speak about a global navigation (Fig. 8). In this case the robot can find the path between two or more points located somewhere in the map. There are many approaches depending on types of obstacles, dimensionality of the space and restrictions for the robot movements. Among the most frequently used are roadmap methods (visibility graphs, Voronoi diagrams, RRT, case-based reasoning, $\mathrm{A}^{*}$ algorithm, etc.) and methods based on a cell decomposition. A common feature of all these methods is the generating of trajectories composed of line segments.

Industrial environment can be classified as difficult due to a wide range of disturbing elements - dust, electric noise, etc. The connection with the human operator can be lost. In these cases the robot should have its own map and find the right way by itself.

\section{Unconventional mechanisms for robots and machine tools}

Even though the high-speed integrated spindles and linear motors make it possible to reach the highest cutting velocities and feed rates for HSC (High Speed Cutting) applications, the practice all utilizations of these parameters is limited by mechanical problems of machines. With respect to these limits of machine tools and robots with conventional serial kinematics, it appears better to use for HSC or high speed manipulation just the machines with parallel or hybrid kinematic structure. These mechanisms are characterized above all by higher stiffness and higher dynamic parameters (thanks to the reduced moving mass). Few years ago the research group at the University of Zilina started to deal with this area. During this period the research group designed some construction concepts of mechanisms based on a PKS (parallel kinematic structure - Figs. 9a and 9b) and different kinds of simulation software (Figs. 10a and 10b) for these types of mechanism [11].

One of the well-known fully parallel manipulator in general is called Hexapod. Hexapod known also as Stewart platform is a multiaxis machine capable of full six degrees of freedom (DOFs) motion plus spindle rotation at the tool head. Among these advantages, higher structural rigidity along with a large payload capability and high speed motions it will be capable for high speed and high accuracy machining or manipulation. The designed machine tool can work in a fully automated regime.

\section{Multi-software platform for multibody systems synthesis}

The problem during the production machine synthesis solving is to find the suitable construction and the corresponding suitable parameters of the mechanism (i.e. materials, dimensions of body shapes and cross-sections, algorithms for the movement controlling, etc.). The parameters must fulfill some functional and technological requirements. These requirements may relate only to the position of the mechanism elements. In this case we call it the geometrical synthesis. In the other cases we must deal with the velocity and acceleration requirements. The methods solving these problems are within the scope of kinematics synthesis.

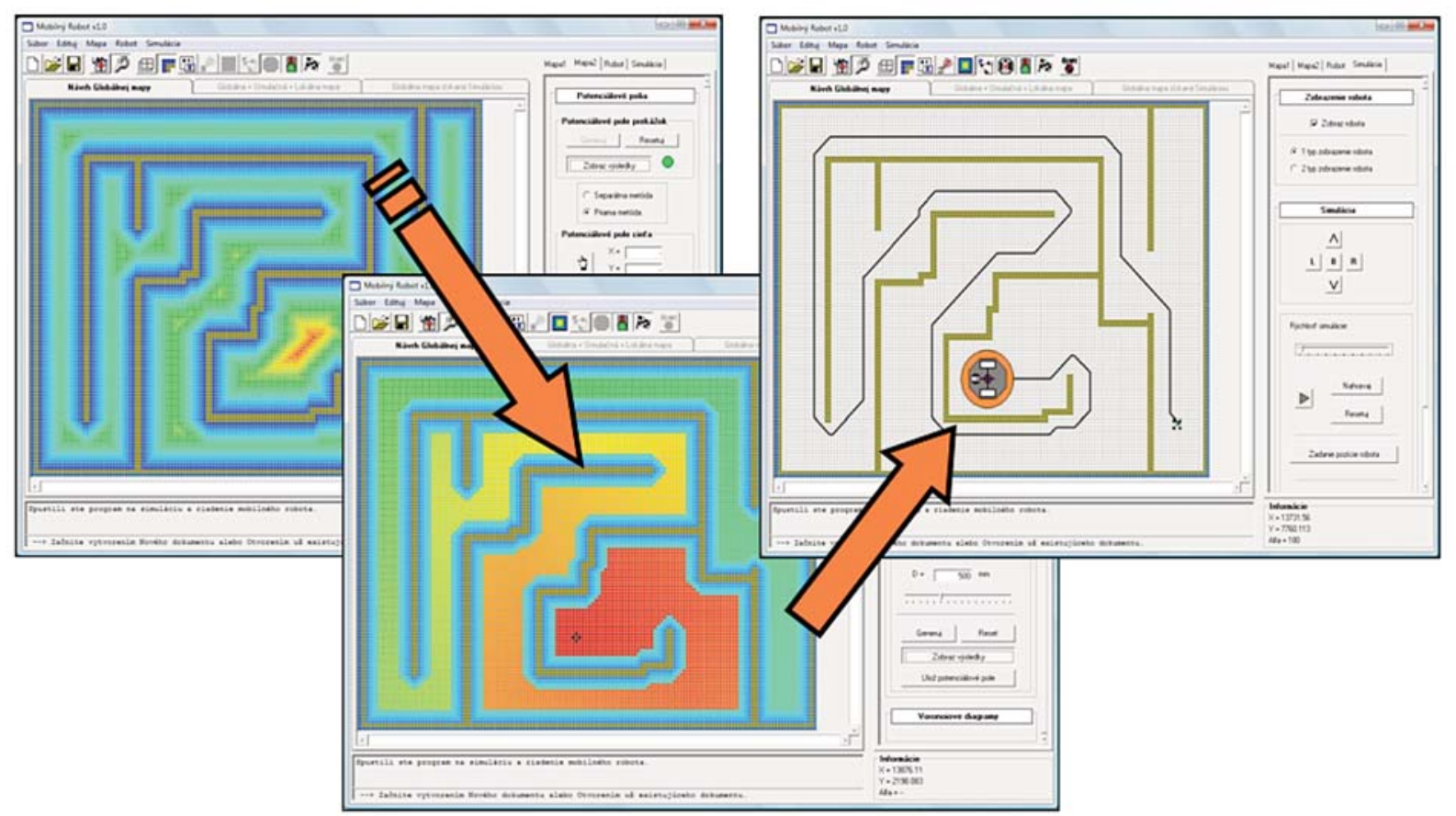

Fig. 8 The method of global navigation (simulation SW Mobile Robot designed at the University of Zilina) 


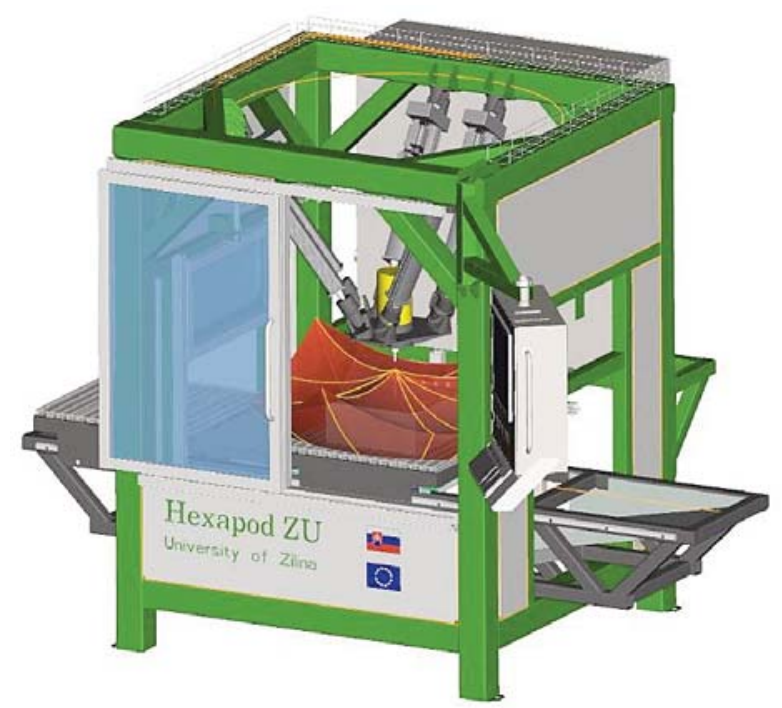

a) The design of machine based on parallel kinematic structure used for high-speed machining or manipulation

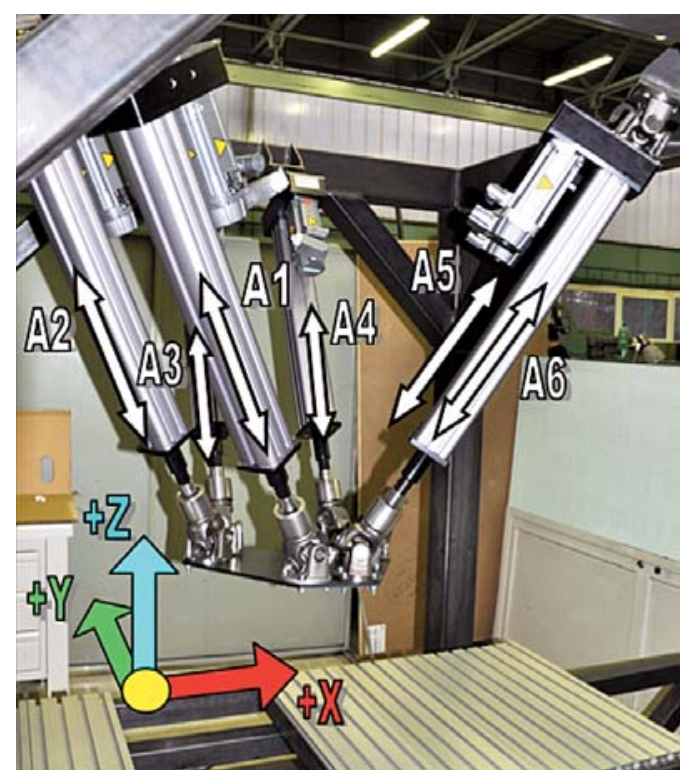

b) The actuators, moving axis (A1 to A6) and Co-ordinate system $(X, Y, Z)$ of hexapod mechanism

Fig. 9 Mechanism with high-speed parallel kinematic structure designed at the University of Zilina [11]

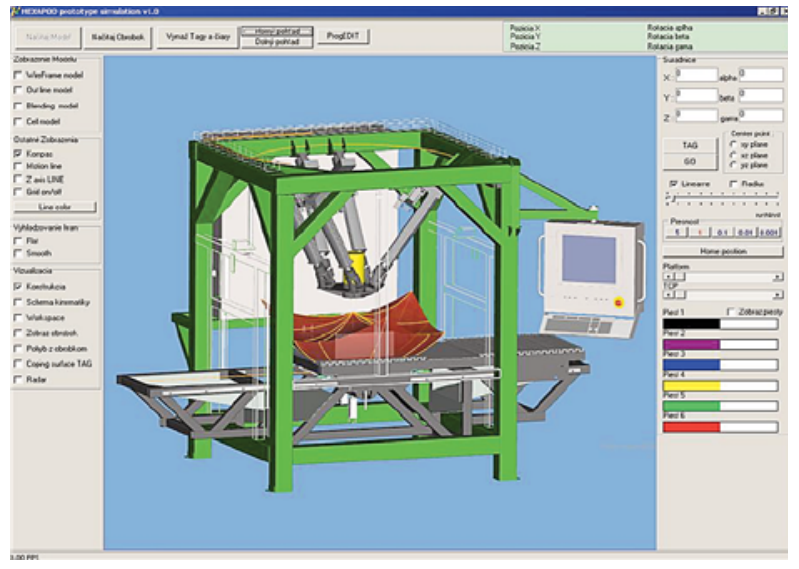

a) Main screen of simulation software designed for control of mechanism with PKS

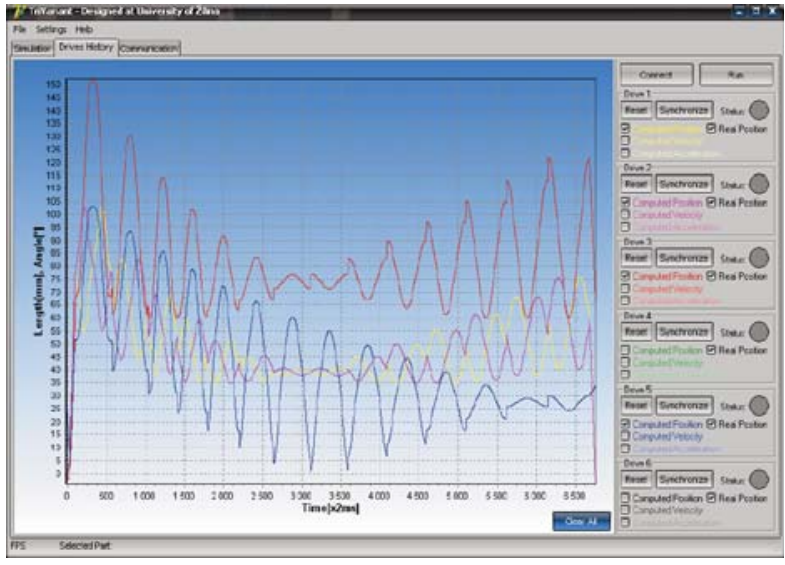

b) The output ramp of acting values obtained from mathematical model of mechanism with PKS

Fig. 10 Simulation software designed for control of mechanism with PKS (University of Zilina)

The dynamical requirements (i.e. balancing, reaction forces reduction, motion irregularity reduction, required motion properties, etc.) are solved by dynamic synthesis [12]. The area of mechanisms synthesis was primarily defined as a relationship between the input and the output (1:1). However, this area is more complicated for the production of machine mechanisms and it covers the whole mechanics and the control area. The solutions can be characterized as multiple inputs - outputs (M:N). Therefore, it is needed to extend the traditional mechanic techniques by the techniques focused on the general design theory [13].

\subsection{Files preparation for the kinematics synthesis of the solid system virtual prototype}

The solution objective is to design and implement the algorithm of the evaluation of the operating gear parameters from the mechanical properties view [14]. The approach is based on the solution of the fixed solid system (FSS) virtual prototypes by means of the computer simulation using the kinematics and optimization [15]. There is tendency to eliminate the time-consuming analyses so that the program interconnection of the ADAMS and MATLAB 
systems will be designed and implemented. For the inverse kinematics problem solving the virtual prototype (VP) manipulator robot was chosen.

\subsection{Virtual prototype preparation in the ADAMS/View environment}

The particular elements of the manipulator robot were designed in the ADAMS/View environment. Kinematic scheme of the manipulating equipment has four movement degrees of freedom (Fig. 11). Quantity " $v$ " is speed of motion and quantity " $\omega$ " is speed of angle.

During the creation of virtual prototypes the models in the ADAMS environment are saved in various file formats according to their purposes. The manipulator robot was created in the binary file format (model.bin). This database contains the information about the working space configuration, all properties of one or more models and the analyses and the simulations results too. The binary format allows the quick writing and loading of the data and it can be transferred between computers with the different operating systems, but it does not allow their reading and editing.

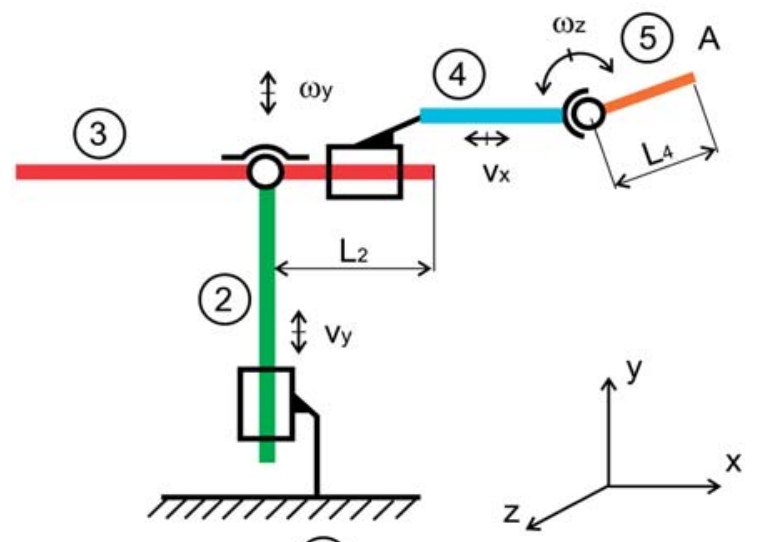

(1)

Fig. 11 Simulation model of industrial robot with serial kinematics

\subsection{Inverted kinematics problem}

The inverted kinematics problem solves the suitable input kinematics parameters multibody of the systems (MBS) for obtaining the described movement [16]. Assuming the stepping motor realistic usage with a step of 5 degrees, all the working positions of the manipulator robot endpoint "A" are mapped (Fig. 12). The point "A" has a screw as the prescribed trajectory. From the obtained working position map the coordinates of those points were chosen which correspond with the prescribed trajectory in the best way and which meet the condition of the continuous movement MBS at the same time.
The input kinematics parameters ensuring the given element prescribed movement MBS are the solution results. Their number depends on the solution step size. Parameter " $r$ "is the minimal distance of the solved point from trajectory. The element lengths were changed from the original $L_{2}=500 \mathrm{~mm}$ and $L_{5}=200 \mathrm{~mm}$ to the optimal ones by the coordinate parametrizing of the markers. The optimal values of the manipulator robot element lengths are: $L_{2}=$ $515.92 \mathrm{~mm}, L_{4}=191.85 \mathrm{~mm}$ (Fig. 11). The genetic algorithm method was used. The objective function value was a numerical zero. In order to obtain the continuous movement the optimal length values was used in the program opt_uhl.m (calculation scheme is shown in Fig. 13).

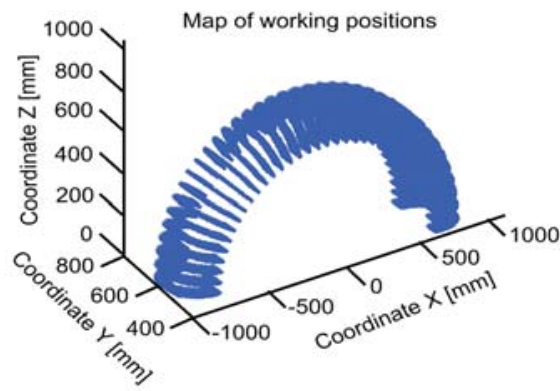

Fig. 12 Point "A" - map of working positions

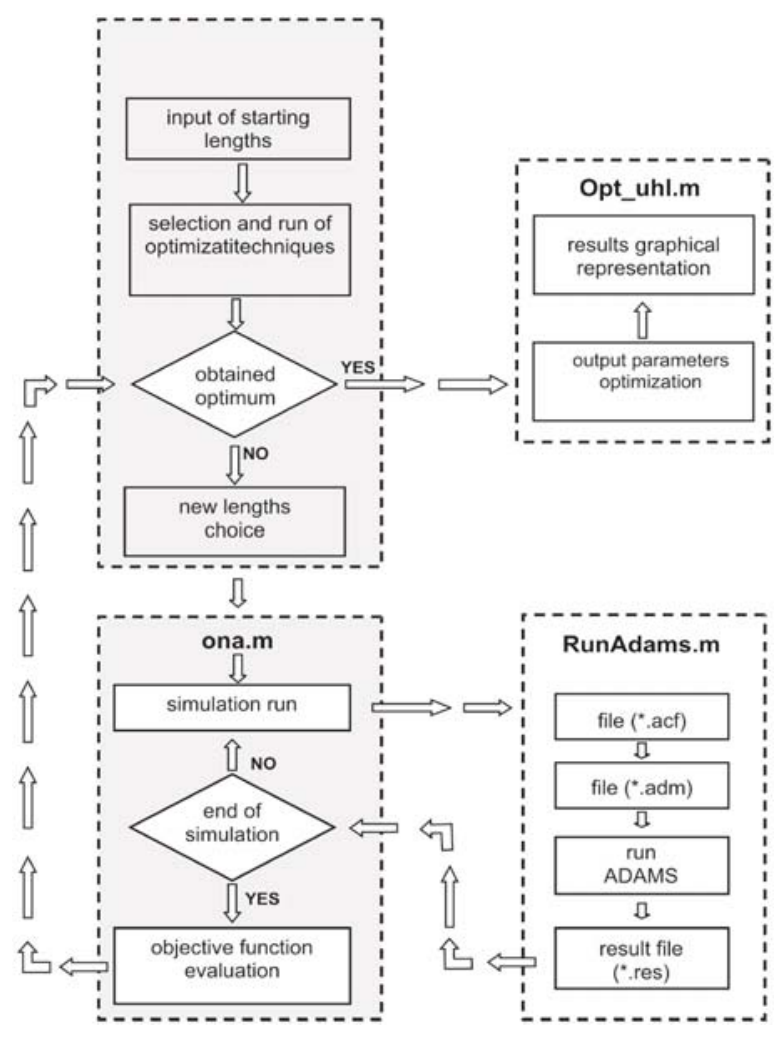

Fig. 13 Graphical representation of the inverted kinematics problem solution 


\section{Conclusions}

The concept of Zilina Intelligent Manufacturing System (ZIMS) covers the basic processes in the entire product lifecycle from beginning to end - PLM (Product Lifecycle Management) as well as PDM (Product Data Management). An example is the design of manufacturing systems by using progressive digital technologies and utilization of automation and robotics in intelligent manufacturing systems and many others. By these progressive technologies the development and product costs can be reduced, product quality and performance improved, and time-to-market reduced too. A very useful tool is a concurrent product design, prototyping and manufacturing, as well as worker training. This approach can be achieved by effective data analysis, visualization, off-line and on-line simulation, advanced interaction and presence within the virtual environment, ergonomics analysis, and collaborative decision-making. The concept ZIMS described in this paper includes all mentioned above.

\section{References}

[1] GREGOR, M., MEDVECKY, S: Application of Digital Engineering and Simulation in the Design of Products and Production Systems. Management and Production Engineering Review, vol. 1, No. 1, pp. 71-84, 2010, ISSN 2080-8208

[2] KURIC, I.: New Methods and Trends in Product Development and Process Planning. In: Academic Journal of Manufacturing Engineering. Editura Politehnica, Scientific Papers, vol. 9, No. 1, 2011, Cluj-Napoca, pp. 83-88. ISSN 1583-7904

[3] HNAT, J.: Virtual Factory Framework. Industrial Engineering Moves the World - InvEnt 2012. Zilina: University of Zilina, 2012. ISBN 978-80-554-0542-1, pp. 56-59, 2012

[4] FURMANN, R., KRAJCOVIC, M.: Modern Approach of 3D Layout Design. In TRANSCOM 2011: 9th European Conference of Young Research and Scientific Workers, Zilina: University of Zilina, 2011, ISBN 978-80-554-0370-0, pp. 43-46

[5] BRUNO, F., CARUSO, F., et al.: Visualization of Industrial Engineering Data in Augmented Reality. J. of Visualization. ISSN 1343-8875, vol. 9, No. 3, pp. 319-329, 2006

[6] MIRANDOVA, G., GABAJ, I., GRZNAR, P.: Use of Augmented Reality in Visual Management. Industrial Engineering Moves the World - InvEnt. Zilina : University of Zilina, 2012, ISBN 978-80-554-0542-1, pp. 112-115.

[7] ENGINEERLIVE - Robotics: Helping Manufacturers Recover. 2011. [online] 2011, [cit. 2012-12-15]. Available on: http://www.engineerlive.com/Process-Engineer/Plant_Management/Robotics\%3A_helping_manufacturers_recover/23205/

[8] FANUC Robotics - The New M1iA. 2009. [online] 2009, [cit. 2012-12-12]. Available on: http://www. fanucrobotics.co.uk/en/countries/fruk/news/the\%20new\%20m1ia

[9] HAVLAS, P.: Riesenie koliznych stavov v oblasti priemyselnych robotov / Solving of Collision Situations in the Field of Industrial Robots), Dissertation thesis. University of Zilina, 2010, p. 139.

[10] URICEK, J., et al.: Control and Navigation of Mobile Robots in Safety and Fire Protection. Communications - Scientific Letters of the University of Zilina, 2011, vol. 13, No. 2, pp. 118-122, ISSN 1335-4205

[11] POPPEOVA, V. et al.: Six-Axes Parallel Mechanism and its Application in Design of Machine Tool with Numerical Control. Scientific Reports, J. of the University of Applied Sciences Mittweida - Moderne Automatisierungtechnik / Robotik, Mittweida, 2011 , vol. 21, No. 5, pp. 74-77, ISSN 1437-7624

[12] SAGA, M., DUDINSKY, M., PECHAC, P.: Optimization of thin Shell Structures Using FSD Algorithms, Communication - Scientific Letters of the University of Zilina, vol. 14, No. 3, 2012, pp. 32-38, ISSN 1335-4205.

[13] SAPIETOVA, A., SAGA, M., NOVAK, P.: Multi-software Platform for Solving of Multibody Systems Synthesis. Communication - Scientific Letters of the University of Zilina, vol.14, No. 3, 2012, Zilina, pp. 43-48, ISSN 1335-4205.

[14] ARNOLD, M., SCHIENHLEN, W.: Simulation Techniques for Applied Dynamics, CISM Courses and Lectures, vol. 507, Italy : Springer Wien : New York, 2008, 313 p. ISBN 978-3-211-89547-4.

[15] SAGA M., HANDRIK M., KOPAS P.: Contribution to Computer Simulation of Induction Bending of Large Diameter Pipes. In. Metalurgija (Metalurgy) 2010, vol. 49, No. 2, p. 498-502, ISSN 0543-5846.

[16] DE JALlON, J. G., BAYO, E.: Kinematic and Dynamic Simulation of Multibody Systems. The Real-Time Challenge, SpringerVerlag : New York, 1994, 440 p., ISBN 0-387-94096-0 